\title{
Developing a neighborhood-scale wireless notification prototype
}

\author{
Sumita Mishra \\ Rochester Institute of Technology \\ sxm1145@rit.edu
}

\begin{abstract}
We outline an innovative approach to the development of a prototype of a neighborhood notification system (NNS). The NNS application residing on smart phones will use software defined radio and cognitive radio components to interface with radio frequency transceivers. Mesh networking is proposed for emergency notification and disaster response coordination using NNS. Our focus has been on the IEEE 802.15.4 and the very recent IEEE 802.15.5 mesh networking standard for low data rate connectivity among low power nodes (or nodes whose power consumption needs to be low). The innovation stems from bringing together different hardware and software components - some of which, like our Software Defined Radio (SDR) platform, are themselves still evolving and others, like the meshing platform, are very new - to propose an adaptive, reconfigurable, infrastructure-less ad hoc wireless solution to emergency communications in the unlicensed ISM RF band.
\end{abstract}

\section{Introduction}

There is growing interest in using wireless technology and networking in innovative ways to relay delay-sensitive information in public safety and disaster recovery emergencies. The Wireless Innovation Forum's recent "Use cases for cognitive applications in public safety communications systems" document is a case in point [1]. In such situations, responders need to be able to use available frequency bands and may not always have a networking infrastructure -- such as WiFI or Internet access - conveniently available to support their communication needs. Emerging new applications of radio technology such as software defined radio and cognitive radio -- dynamic spectrum access (DSA) technologies in general - figure prominently in scholarly discussions of innovative solutions to emergency situations. The wireless grids approach [2] and Virginia Tech's software-defined radio /cognitive radio test-bed called OSSIE/CORNET [3] represent a complementary initiative to develop the software components and interfaces necessary to enable adaptive communications systems for a broad range of uses over diverse radio frequency (RF) hardware front-ends. At the physical and media

\author{
Murali Venkatesh, Bahram Attaie \\ Syracuse University \\ \{mvenkate, battaie@syr.edu\}
}

access control (PHY/MAC) layers, there is a corresponding high level of interest in developing specifications for infrastructure-less neighborhoodscale ad hoc mesh wireless networking technologies, as evidenced by the IEEE Working Groups that are very active around the IEEE 802.15.3, .4, and .5 specifications. In this paper, we present a preliminary report on the development of a Neighborhood Notification System (NNS) building on software defined radio and the recently-released IEEE 802.15.5 mesh networking specifications for lowpower nodes [4]. Our work on the NNS, which is ongoing, is undertaken under the aegis of the Wireless Grids Innovation Test-bed (WiGIT) at Syracuse University [5].

The work we report here uses concepts from adaptive radio. Adaptive radio systems or devices have the ability to monitor their own performance and provide the means of "varying their own parameters by closed-loop action to improve their performance" without human intervention [1]. Within the umbrella of adaptive wireless communications systems, we specifically use a software defined radio (SDR) and cognitive radio (CR) test-bed platform developed by Virginia Tech [3]. The term "software defined radio" refers to a class of technologies wherein some or all of the functions traditionally implemented in the PHY/MAC layers are defined in software. The term "software defined" refers to the use of software processing within the communications device to implement the operating functions - including the frequency range(s) and modulation types(s) supported, and output power [6]. Cognitive radio is an encompassing concept and denotes an operating environment consisting of software-defined devices and other communications technologies with an ability to adapt to operating conditions without human intervention.

The Software Communications Architecture (SCA) has gained a fair degree of industry support as a framework and platform for SDR work [7]. It is not the only such platform, but we selected it for our purposes because of its availability as a test-bed at Virginia Tech and the knowledge base that researchers there have built up on the platform over the years. The SCA resulted from research underwritten by the US Department of Defense for the specification of an architectural framework to

DOI: 10.5176_2010-2283_1.2.63 
promote research and development of adaptive reconfigurable joint tactical radio system (JTRS) SDR applications. The SCA is designed to support modular applications software ("waveforms") to be assembled from multiple, reusable, modular signal processing units that inter-communicate using the language and platform-independent Common Object Request Broker (CORBA) middleware architecture. A "waveform" refers to the complete set of applications software that together support end-toend communications between end systems on a network [7].

A key premise of SDR is its flexibility and adaptability to operating conditions via reconfigurability. We believe that these characteristics promised by SDRs and CRs are a key aspect of their suitability in emergency situations and in disaster sites. Apropos [1], the Wireless Innovation Forum's (2010) recent publication calls attention to the ability of CR technology to make "real-time adjustments... with little or no human intervention" and the conviction that such a capability, "residing in radios or implemented in a network...can improve the communications of public safety first responders". Software radios offer several advantages over conventional communications: multifunctionality from reconfigurability, global mobility from compatibility with popular world-wide standards such as WiFi, physical compactness from the fact that the same hardware may be used to implement more than one radio design, and ease of upgrade from their dynamic interface capabilities [6]. Cognitive radios add dynamic, on-the-fly, policydriven automated adaptability and configurability to these advantages. Our work relies on SDRs since the system - intended for notification and communication on the scale of an urban neighborhood -- we are currently prototyping would need to be adaptive and flexible using available wireless means (WiFi, WiMax, mesh-capable IEEE 802.15.3 and .4) in order to be able to establish and sustain reliable communications in emergency situations and in disaster sites.

This paper is organized as follows. We start with the WiGIT test-bed and the NSF-funded Wireless Grids project that motivates it. A brief overview of the IEEE 802.15.4 and .5 technologies follows. The neighborhood notification system (NNS) use case is then outlined. Our focus on neighborhood-scale communications systems - envisaged as covering one or two city blocks - motivates what we are calling a wireless NAN - or neighborhood area network, an ad hoc network of mesh-capable WPANs fitting in between a WPAN and a WLAN in terms of its operational range. Notification, communications, and disaster recovery efforts tend to occur over focused areas around the physical disaster site, and we believe that the IEEE's new mesh networking specifications described in the 802.15.5 standard [8] provide a robust basis for developing applications spanning multiple meshed wireless PANs for operation in a neighborhood and supporting both low-rate and highrate data communications. We then present our approach to developing a prototype NNS, with the focus in this paper being on the Phase 1 work: lowrate communications using the IEEE 802.15.4 specifications for WPANs and wireless sensor networks and the IEEE 802.15.5 mesh networking standards.

\section{WiGIT: The Wireless Grids Innovation Test-bed}

The WiGIT test-bed builds on wireless grids [2]. The wireless grids project - named a "transformative innovation" by National Science Foundation (NSF) -has enjoyed decade-long support from the NSF Partnership for Innovation (PFI) program, leading research universities and corporations, foundations, and state government agencies. The wireless grids initiative is driven by the conviction that wireless devices should be able to seamlessly connect to and inter-operate with other proximate devices, of whom they have no prior knowledge, for accessing resources, which could include CPU sharing, as well as applications and data resources sharing. The devices thus connected could be diverse, in other words heterogeneous. The software enabler of ad hoc connectivity among proximate heterogeneous devices is called the "edgeware". Edgeware is an application layer overlay designed to enable "ad hoc connection of people, devices, software and services in a personal cloud, supported by personal cyberinfrastructure" [5]. SDR and CR capabilities, when fully incorporated into WiGIT will allow edgeware applications to access and use a wide range of wireless communications technologies. Building on the SDR and CR capabilities, edgeware applications "can dynamically make use of content and resources present in devices... and determine how to efficiently use the resources based on environmental and user/application constraints and preferences" [5].

WiGIT is an ongoing initiative with continued support from the NSF, Syracuse University and Virginia Tech (VT), and partners from MIT, Tufts University and Instituto Superior Tecnico (Lisbon, Portugal). WiGIT is intended as a "research playground" (or test-bed) where academic researchers work closely with their counterparts in industry to develop and test open API specifications 
for wireless grids. The wireless grids application programming interfaces (WGAPIs), when fully developed, will implement the group's strategic goal of enabling ubiquitous connectivity for edgeware applications. Specifying an open interface to SDR technologies is part and parcel of this effort, which is directed at the bottom end of Figure 1 below. Other areas for WiGIT R\&D include "application performance and optimization, characterization of networks for wireless grid applications, and protocol development. Additionally, user behavior trials, application tests, security models, and trust frameworks for wireless grids will be among the issues explored..." [5]. Additional details on WiGIT can be found at (http://wglab.net).

Figure 1 below shows WiGIT's cognitive radio elements in the context of the Virginia Tech CORNET test-bed. The OSSIE framework developed by Virginia Tech represents the SDR platform in the figure. In contrast to WiGIT's ongoing work on developing edgeware applications and specifying open WGAPIs, which address opportunities for innovation at upper layers of the stack, wherein the edgeware for example accesses the CR shell over the WGAPIs, our NNS work is mostly at the lower layers at the present time, specifically at the PHY/MAC layers of the SDR - RF front-end. This is so given the project objectives to provide a means for local connectivity over the unlicensed ISM wireless band whether it is WiFI, WiMAX, or newer technologies like wireless sensor networking. Further, SDRs generally do not provide PHY/MAC-level details, leaving these up to developers. Additionally, the OSSIE implementation at Virginia Tech (using the universal software radio peripheral, or USRP) targets laptops as the end device and $\mathrm{WiFi}$ as the communications technology.We extend this work, as well as the work of WiGIT more generally, by exploring 802.15.5 and related specifications with smart phone as the end devices.

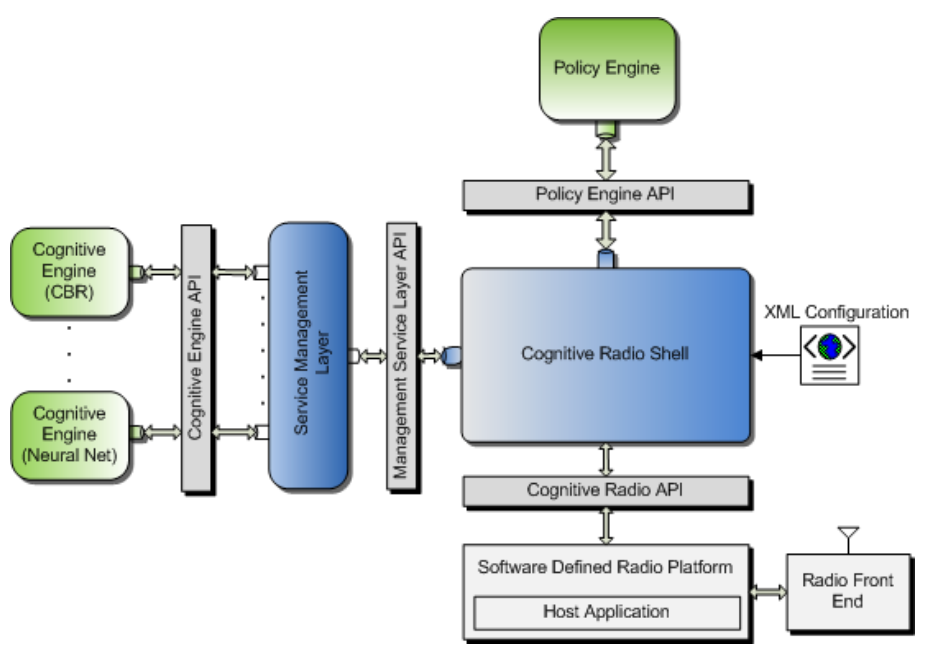

Figure 1: Cognitive Radio Elements for WiGiT (from [3])

\section{Meshing ad hoc networks for low rate (LR) communications}

The IEEE's 802.15.4 specifications cover wireless connectivity for devices such as sensors. These specifications - offered as an enabling standard complementing other standards like the 802.11 (WiFi) and ultra wideband (UWB) - are intended for devices with certain distinctive characteristics, including low-power consumption, low data rate, low cost, and restricted operational range in the tens of meters.
The 802.15.4 specifications define the PHY and MAC layers for low data rate transmission $(250 \mathrm{~Kb} / \mathrm{s}$ or less). Such networks are LR-WPANs, or low rate wireless personal area networks. LR-WPANs support technologically simple devices that use minimal power and typically operate in the personal operating space of $10 \mathrm{~m}$ or less. The standards cover two topologies: a one-hop star or, when lines of communication exceed the $10 \mathrm{~m}$ operating span, a multi-hop peer-to-peer topology. The 802.15.4 specifications, released in 2003, also cover wireless sensor networks. ZigBee, a WPAN/WSN technology promoted by an industry alliance, shares some of these features. Notable characteristics of the IEEE 
802.15.4 specifications include the following. More details are available at [4].

1. This standard defines two PHY layer specs: one operating in the unlicensed $2.4 \mathrm{GHz}$ industrial, scientific and medical (ISM) band, which is available worldwide, and the other in the ISM $868 \mathrm{MHz}$ and $915 \mathrm{MHz}$ bands available in Europe and North America.

2. At the MAC sub-layer, the standard supports unslotted CSMA-CA and slotted CSMA-CA and non-beacon-enabled and beacon-enabled mode. The beacon-enabled mode is recommended for greater power-saving in LR-WPANs.

3. As many as 27 channels with three different data rates are specified, ranging from 16 channels with a data rate of $250 \mathrm{~Kb} / \mathrm{s}$ to one channel of 20 kbps in the $868 \mathrm{MHz}$ band. An LR-WPN can choose to work in one of these 27 channels depending on availability, congestion, and the channel's data rate.

4. Two different types of devices are supported in an LR-WPAN: An FFD and an RFD. The FFD, or full function device can communicate with all other FFDs and RFDs on the network, while an RFD can only communicate with an FFD. Besides, an FFD can serve as the PAN coordinator, coordinator, or device; an RFD can only operate as a device.

5. Data transfers between nodes can occur in three different ways: from a device to a coordinator, from coordinator to a device, and from one peer to another in a peer-to-peer multi-hop network.

The release of the IEEE 802.15.5 specifications (in 2009) is a significant development in WPAN communications and connectivity [4]. The purpose of the specifications, which are offered as a Recommended Practice by the IEEE, is to "provide the architectural framework enabling WPAN devices to promote interoperable, stable, and scalable wireless mesh topologies and, if needed, to provide the amendment text to the current WPAN standards that is required to implement this recommended practice". The IEEE 802.15.5 specifications recommend a mesh sub-layer on top of the existing 802.15.x PHY/MAC layer protocols (the 802.15.3 protocols for high data rate and 802.15.4 protocols for low data rate communications). By supporting multi-hop routing, the mesh sub-layer would enable mesh-capable WPANs to overcome the physical coverage limitations associated with single WPAN topologies generally while also providing for greater reliability via route redundancy.
Lee et al. report the successful testing of the meshing function implemented in a sub-layer [4]. This mesh sub-layer, located between the MAC sub-layer and the service specific convergence sub-layer (SSCS), is developed using the 802.15.4 PHY/MAC stack on the TI CC2420 chipset. The CC2420, which is a IEEE 802.15.4 compliant RF transceiver designed to work in the $2.4 \mathrm{GHz}$ ISM band for low power and low voltage wireless applications, provides a data rate of $250 \mathrm{~Kb} / \mathrm{s}$. The chipset is part of the Micaz sensor board operating on the TinyOS platform.

\section{The Neighborhood Notification System (NNS) Project: Background}

Currently, the focus of development of WiGIT APIs is on the IEEE 802.11 (WiFi) specifications, with the Virginia Tech SDR platform OSSIE (Open-Source SCA-compliant Implementation: Embedded) serving as the component in between the WiGIT edgeware wrapper at the application layer and the RF hardware front-end. The Neighborhood Notification System uses the OSSIE SDR software platform and the Universal Software Radio Peripheral boards for prototype development and testing. USRP board was developed by Ettus Research for the GNU radio community.

OSSIE [3] is intended as an open-source extensible environment for the development of waveforms under the umbrella of the SCA - the Software Communications Architecture - in the joint tactical radio systems (JTRS) program of the US Department of Defense. As noted earlier, the SCA offers a framework for SDR work. The JTRS initiative seeks to smooth radio inter-operability problems by providing developers a robust set of programmable, easily-extensible, multi-mode, multiband, and modular radio applications for use in diverse RF environments. The SCA specifies the design, development and management guidelines for wireless communications systems consistent with the aims of the JTRS program. Despite its DoD origins, the SCA has been found to be useful for commercial and nonmilitary research and development in SDRs as well. The OSSIE platform is written for Windows 2000 and Linux (Fedora) in $\mathrm{C}++$ using the omniORB and Xerces XML parser. Python and wxPython for mobile applications development are supported.

The USRP is an integrated hardware board which incorporates very simple functionality. The basic architecture consists of a motherboard and up to four daughterboards for basic RF transmit and receive functions. The USRP connects to a PC, laptop or other device over the USB2 interface (or even USB 
1.1 with appropriate modifications). The board provides AD/DA converters, one or more daughterboards, (the RF front-end), and a field programmable gate array (FPGA) that inter-links these elements (and the host USB interface) and implements general purpose operations including digital conversion, decimation, and interpolation (5). The schematic below shows the USRP with four analog to digital converters (ADCs). The FPGA is an Altera Cyclone EPIC12, which connects to a USB2 interface chip (the Cypress FX2) on the device. The FPGA and the USB microcontroller are programmable over the USB2 bus.

Recent developments in SDR research improve on some of the SCA's limitations with respect to ease of automated on-the-fly reconfigurability in dynamic use environments. Typically, carrier frequency can be varied in most radios. SDR applications may require reconfiguration of other parameters as necessary, such as modulation scheme used, as exemplified by $\mathrm{AM} / \mathrm{FM}$ radio receivers that can work in either modulation scheme. The example is readily extended to digital modulation schemes and devices (Cormier et al., 2010). In fact, in the SDR and CR approaches, recall that such flexibility and adaptability, as well as the receiver's ability to interpret, reconfigure and deploy the appropriate policies as the operating environment demands, are a key premise. Recently, Cormier et al. (2010) reported a SDR design for automated dynamic reconfiguration, improving on the SCA's support only for static configuration and infrequent changes to the initial configuration.

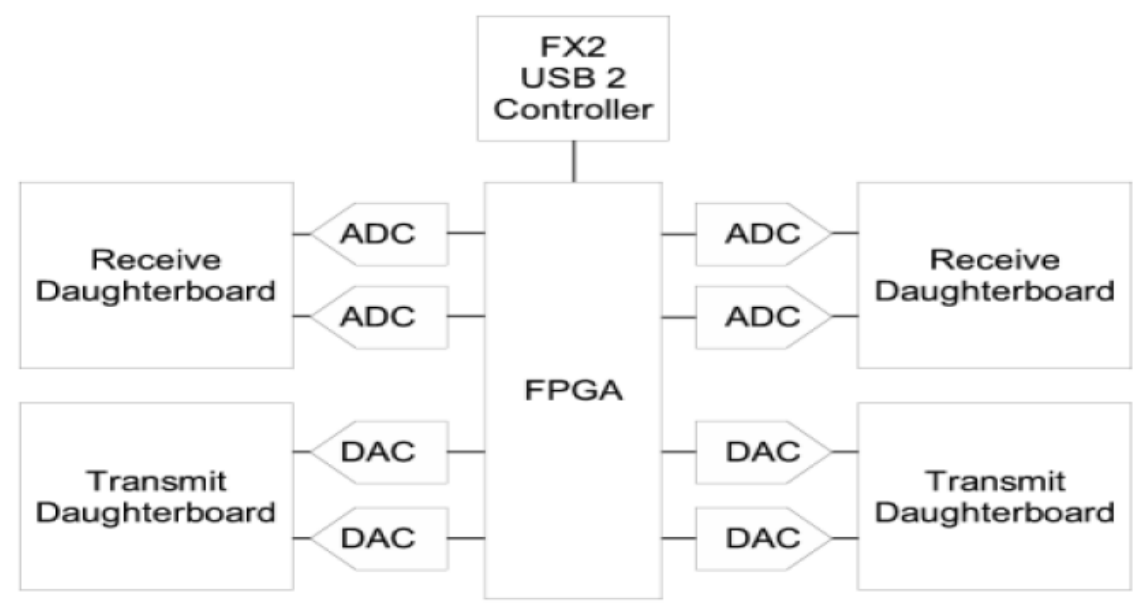

Figure 2: USRP board schematic (from [6]).

\section{Project Rationale and Objectives}

The objective of the NNS project is to develop and test a prototype system for use in an urban neighborhood spanning a few city blocks. The system is envisioned as an infrastructure-less, meshed, ad hoc network connecting a number of low-power devices. In Phase 1, our aim is to work with sensors (such as the CC2420 chipset and Micaz), with the "sink node" being a laptop. The OSSIE platform/USRP board will serve as the intermediate component between the sensor and the sink node, which in this case will be the smart phone (however, initially, we envision the sink node being a PC laptop). The Phase 1 work will focus on developing a prototype using sensors for low data rate (250 Kbps or less) communications on the meshed network.

\section{NNS Phase 1 Use Case}

Xerxes Biomass (pseudonym) is a fast-growing startup in the green energy conversion business. Their many processing and conversion systems (such as the biomass grinders) need constant monitoring over the whole course of the production cycle. The NNS project team has worked with Xerxes in the Fall 2010 semester to develop a plan for a prototype installation of a meshed WSN-based process monitoring and control system using the IEEE 802.15.4 and 802.15.5 specifications implemented on Micaz/CC2420/ TinyOS platform, with a laptop being the sink node. The test-bed installation reported in Lee et al., (2010) will be a key resource and exemplar as we undertake this work. The IEEE 802.15.4g working group's efforts in the area of utility smart grid communications as an extension of the 802.15.4 
specifications will also be examined for future development of prototype functionality for Xerxes Biomass. This Phase 1 work will continue as long as necessary until testing of the prototype installation is completed. After the initial phase of testing and as part of the Phase 1 work, integrating the prototype with the OSSIE SDR platform and a smart phone as sink node will be examined for feasibility. The Center for Excellence (CoE) for green energy initiatives at Syracuse University supports other startups/projects like Xerxes, so the lessons learned in Phase 1 is expected to be of value beyond the proximate use-case.

\section{NNS Phase 2 Use Case}

The idea for an NNS started out as a way to solve a problem brought to us by neighborhood leaders on Syracuse city's south side. They were looking for a technology that would allow them to share information on an emerging crisis in the neighborhood so that they could mobilize quickly to defuse it. Since then, other potential uses for such a system have emerged. For example, ethnic Kirin refugees from Burma who have been resettled in the city's north side want an inexpensive communications system. Members of the local deaf community have voiced a need for an affordable communications system with neighborhood coverage with possibly a GPS-based location reporting feature. In Phase 2, the NNS project team intends to develop a multi-mode, meshed, ad hoc neighborhood area network using the IEEE 802.15.3 (if the hardware is available in the marketplace) and the IEEE 802.15.5 specifications to support high data rate transmission and QoS over multiple mesh-capable WPANs [4], with Nokia Symbian smart phones as the devices.

\section{Conclusion}

Extended goals of the NNS project are two-fold. As noted earlier, through this work we hope to contribute to the development of open specifications for adaptive, reconfigurable, infrastructure-less ad hoc WNANs - wireless neighborhood area networks, i.e., networks whose operating range is a typical urban neighborhood of two city blocks. The appeal of such WNANs for emergency notification and disaster response are readily apparent. The second extended goal is to develop the Virginia Tech OSSIE SDR platform itself as a Java-based platform so as to support the development of the NNS application and possibly other edgeware applications - on the Android OS for smart phones.

\section{References}

[1]. Wireless Innovation Forum (2010). Use Cases for Cognitive Applications in Public Safety Communications Systems Volume 2: Chemical Plant Explosion Scenario. Document WINNF-09-P-0015V1.0.1.

[2]. McKnight, L., Howison, J., and Bradner, S. (2004). Wireless grids: Distributed resource sharing by mobile, nomadic, and fixed devices. IEEE Internet Computing, July/August, 24-31.

[3]. OSSIE (2010). SCA-based Open Source SDR VirginiaTech, http://ossie.wireless.vt.edu/

[4]. Lee, M.J., et al (2010). IEEE 802.15.5 WPAN Mesh Standard-Low Rate Part: Meshing the Wireless Sensor Networks. IEEE Journal on Selected Areas in Communications, 28 (7).

[5]. McKnight, L., Caicedo, C., Ramnarine-Rieks, A., and Treglia, J. (2010). Towards open Wireless Grids: Personal cyberinfrastructure specification by innovation test-bed. Unpublished mss. Wireless Grids Innovation Test-bed, Syracuse University.

[6]. Hasan, S.M.S., and Balister, P. (2005). Prototyping a SDR receiver based on USRP and OSSIE. Chameleonic Radio Technical Memo, Bradley Department of Electrical and Computer Engineering, Virginia Tech.

[7]. Cormier, A.R., et al (2010). Dynamic reconfiguration of software defined radios using standard architectures. Physical Communication, 3.

[8]. IEEE Computer Society (2009). Part 15.5: Mesh Topology Capability in Wireless Personal Area Networks (WPANs). New York, NY: IEEE.

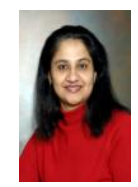

Sumita Mishra, Ph.D., is Assistant Professor at Rochester Institute of Technology in the Networking, Security and Systems Administration department. Her current research involves security solutions for ad hoc, sensor and smartgrid networks. Dr. Mishra has published over 40 scientific papers in international conferences and journals.

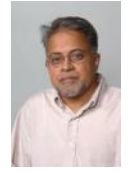

Murali Venkatesh, Ph.D., is Associate Professor at the School of Information Studies, Syracuse University. His research and teaching interests include data communications and e-gov systems.

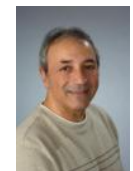

Bahram Attaie is a Professor of Practice at Syracuse University's School of Information Studies (iSchool). He oversees the research activities of the unified communications test bed located in the school's Center for Convergence and Emerging Network Technologies (CCENT). He is currently teaching advanced topics in networking at graduate and undergraduate levels. 